\title{
Unter den Diabetikern sind Immigranten gesünder als Einheimische
}

Fragestellung: Unterscheiden sich Immigranten mit Diabetes mellitus, die in westliche Industrienationen ziehen, langfristig hinsichtlich der Häufigkeit kardiovaskulärer Komplikationen und der Sterblichkeit von Einheimischen mit Diabetes?

Hintergrund: In den letzten Jahrzehnten hat die Lebenserwartung in den westlichen Industrienationen deutlich zugenommen, während sich die gesundheitliche Situation in Ländern mit geringem und niedrigem Wohlstand verschlechtert hat. Heute ziehen nun immer mehr Menschen aus uns fremden Ländern und Kulturen in die hoch

\section{Originalie}

Okrainec K, Bell CM, Hollands $S$ et al. Risk of cardiovascular events and mortality among a population-based cohort of immigrants and long-term residents with diabetes: Are all immigrants healthier and if so, for how long? Am Heart J

2015;170:123-32 entwickelten Länder. Bislang ist unklar, ob bei den Immigranten Änderungen des Lebensstils und Probleme der Eingewöhnung und Anpassung den Gesundheitsstatus verschlechtern oder verbessern.

Patienten und Methoden: Die Autoren haben die Daten von Diabetikern, jeweils 87.707 Kanadier und Immigranten aus diversen Ländern, vorwiegend aber aus Asien und Afrika, überprüft. Letztere mussten schon seit mindestens 3 Jahren in Kanada leben ( $65 \%$ schon seit $>10$ Jahren). Für diese beiden Kohorten wurden von 2005 bis 2012 aus dem nationalen Register alle kardiovaskulären Komplikationen (Herzinfarkte, Herzinsuffizienz, instabile Angina pectoris und Schlaganfälle) sowie alle Todesfälle entnommen.

Ergebnisse: Kardiovaskuläre Komplikationen und Todesfälle waren bei Langzeit-Kanadiern signifikant häufiger als bei Immigranten (3,5 bzw. 2,5 pro 100 Jahre). Auch unter Berücksichtigung vieler Einflussfaktoren wie Alter, Geschlecht, sozialer Status, Begleiterkrankungen und Zugang zu medizinischer Versorgung war der Unterschied schon nach einem Jahr erkennbar und persistierte über die gesamte Beobachtungsdauer. Kardiovaskuläre Komplikationen und Todesfälle waren bei Einheimischen um $24 \%$ signifikant häufiger.

Schlussfolgerung: Diabetiker, die nach Kanada einwandern, haben ein geringeres Risiko für kardiovaskuläre Komplikationen und Tod als Langzeitkanadier mit Diabetes.

\section{- Kommentar von Prof. Dr. med. Heinrich Holzgreve}

\section{Gesunde kommen, Kranke gehen}

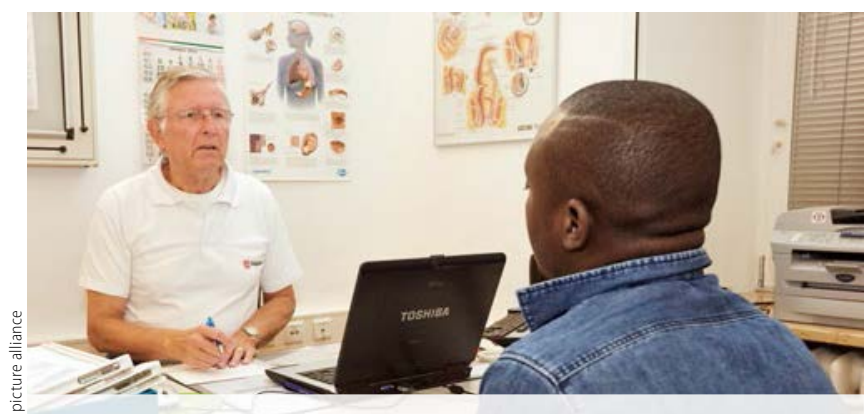

Brauchen Immigranten seltener einen Arzt als Langzeit-Deutsche?

Schon 2008 berichtete das Bundesamt für Migration und Flüchtlinge, dass die Sterblichkeit von erwachsenen Immigranten geringer ist als bei erwachsenen Deutschen. Die vorliegende Untersuchung aus Kanada erweitert diese Beobachtungen auf Diabetiker und auf deren kardiovaskuläre Komplikationen und die Mortalität.

Der Gesundheitsvorteil diabetischer Immigranten gilt kurzund langfristig, d.h. im ersten und zweiten Jahrzehnt nach der Einwanderung, für die Mehrzahl von Untergruppen. Nur eine
Minderheit, nämlich Flüchtlinge, neue Einwanderer aus bestimmten Regionen (Osteuropa, Zentralasien, Lateinamerika, Mexiko, Afrika südlich der Sahara, Westeuropa und den USA), ferner Personen mit geringer Bildung und ohne soziale Hilfe zeigten in den ersten Jahren nach der Einwanderung nur einen geringen, nicht signifikanten Gesundheitsvorteil.

Da zahlreiche Einflussfaktoren berücksichtigt wurden, gibt es nur eine Erklärung für diesen Befund: Einwanderer, die die Umsiedlung auf sich nehmen, sind eine Selektion gesünderer Menschen (Healthy migrant Effekt) und möglicherweise kehren Migranten, wenn sie ernsthaft erkranken, „wie Lachse zum Laichen" in ihr Heimatland zurück (Salmon-bias-Effekt).

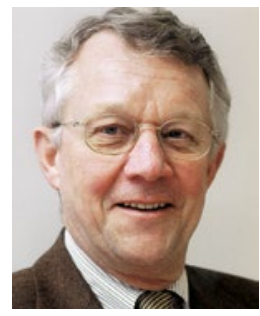

Prof. Dr. med. Heinrich Holzgreve

Internist, Kardiologische Praxis

Burgstr. 7, 80331 München

h.holzgreve@t-online.de 